\title{
Demographic and Assisted Reproduction Related Factors Associated with Dichorionic Triplet Gestations
}

\author{
Barbara V. Parilla ${ }^{1}$, Brett Goldman², Sue Jasulaitis ${ }^{3}$, Suela Sulo ${ }^{4}$, Angeline Beltsos ${ }^{3}$ \\ ${ }^{1}$ Department of Obstetrics and Gynecology, Advocate Lutheran General Hospital, Park Ridge, USA \\ ${ }^{2}$ Chicago Medical School, North Chicago, USA \\ ${ }^{3}$ Fertility Centers of Illinois, Chicago, USA \\ ${ }^{4}$ James R. \& Helen D. Russell Institute for Research \& Innovation, Advocate Lutheran General Hospital, Park \\ Ridge, USA \\ Email: "Barbara.Parilla@advocatehealth.com
}

Received 25 September 2015; accepted 7 November 2015; published 10 November 2015

Copyright (C) 2015 by authors and Scientific Research Publishing Inc.

This work is licensed under the Creative Commons Attribution International License (CC BY).

http://creativecommons.org/licenses/by/4.0/

cc) (i) Open Access

\begin{abstract}
Our objective was to evaluate which demographic factors or assisted reproductive technologies were associated with IVF triplet gestations where one of the embryos split, resulting in a dichorionic triplet gestation. This was a case-control study of dichorionic versus trichorionic triplet gestations that underwent assisted reproductive technology over the last 5 years at our fertility center. There were 53 cases of dichorionic triamniotic triplet gestations compared to 119 trichorionic triplet controls. There were no significant demographic differences between the cases and controls. 51/53 dichorionic triplets and 86/119 trichorionic triplets were conceived through IVF, the remaining utilized intrauterine insemination. ICSI was performed in virtually all patients that underwent IVF. Of the potential risk factors studied, hatching was used in $70.6 \%$ of dichorionic compared to $89.5 \%$ of trichorionic IVF triplets $(p=0.005)$; embryo transfer was performed on Day 5 or 6 compared to Day 3 in $\mathbf{8 8 . 0 \%}$ dichorionic vs $\mathbf{7 1 . 8 \%}$ trichorionic $(\mathbf{p}=\mathbf{0 . 0 2 8}$ ). Frozen sperm was utilized more frequently with dichorionic than with trichorionic triplets, $26.0 \%$ vs $10.9 \%$ (p < 0.011). Only $4(7.5 \%)$ of the IVF cases underwent pre-implantation genetics. Certain assisted reproductive technologies appear to be associated with embryo splitting and a dichorionic triplet gestation. More research is needed in this area to further elucidate these findings.
\end{abstract}

\section{Keywords}

Monochorionic, Triplets, Embryo Splitting

\footnotetext{
${ }^{*}$ Corresponding author.
}

How to cite this paper: Parilla, B.V., Goldman, B., Jasulaitis, S., Sulo, S. and Beltsos, A. (2015) Demographic and Assisted Reproduction Related Factors Associated with Dichorionic Triplet Gestations. Open Journal of Obstetrics and Gynecology, 5, 740-745. http://dx.doi.org/10.4236/ojog.2015.513104 


\section{Introduction}

The incidence of multiple gestations has risen significantly over several decades, primarily due to the increased use of fertility drugs for ovulation induction, superovulation, and assisted reproductive technologies (ART) such as in vitro fertilization (IVF). In 1960, before the commercial availability of drugs for the treatment of infertility, there were approximately 4.3 million births in the United States, with 1244 triplets and higher order multiples. In 2011, the total number of births was slightly lower (3.9 million), but there were 5417 triplet and higher order multiples. Approximately $20 \%$ of high order multiple births are naturally conceived; $39 \%$ to $67 \%$ are related to ovulation induction/superovulation; and $13 \%$ to $44 \%$ are associated with ART.

Multiple gestations are at significantly increased risk of fetal, neonatal, and maternal complications, as well as complete pregnancy loss, when compared to singleton pregnancies [1]-[3]. As an example, in one review, the risk of delivery less than 32 weeks of gestation for singleton, twin, triplet, and quadruplet pregnancies was $2 \%$, $8 \%, 26 \%$, and greater than $95 \%$, respectively [4].

Fertility centers aim to balance success along with limiting higher-order multiples. However, even with the transfer of 2 embryos, there appears to be an increasing number of triplet gestations with a monochorionic twin pair. This occurs when one of the transferred embryos splits into a twin pair that shares a placenta.

Monochorionic twin pairs suffer further, additional risks of growth discordancy and twin-twin transfusion syndrome as compared to dichorionic twin gestations. This translates into even earlier preterm delivery rates with increased morbidity, mortality, and long-term sequelae of prematurity as well as total pregnancy loss [5]. This trend is also noted in higher-order multifetal gestations [6]; for example, a triplet gestation that has a monochorionic twin pair is at higher risk of complications than a triplet gestation that is trichorionic [7].

There are only a few case reports in the literature that describe this phenomenon of a monochorionic twin pair in a triplet gestation after the IVF transfer of 2 or more embryos [8]-[11]. Some factors that may predispose to this further split are days of transfer. IVF embryo transfers are usually performed on Day 3 (55\%) or Day 5 (36\%). It has been suggested that there can be as high as a $5 \%$ monochorionic diamniotic rate if the transfer occurs on Day 5 at the blastocyst stage. In addition, ICSI (intra cytoplasmic sperm injection) may also increase this risk.

We aimed to evaluate which demographic factors or assisted reproductive technologies were associated with dichorionic triplet pregnancies.

\section{Materials and Methods}

This was a case control study performed through a retrospective review of a prospectively maintained database from the Fertility Centers of Illinois. A total of 119 trichorionic triplets and 53 monochorionic triplets were identified between January 2009 and June 2014. Cases were designated as patients with a pregnancy consisting of 3 fetal heartbeats and 2 gestational sacs identified by early ultrasound (dichorionic). Controls were designated as patients with a pregnancy consisting of 3 fetal heartbeats and 3 gestational sacs identified by early ultrasound (trichorionic). GE Logiq ultrasound machines with a $4 \mathrm{MGz}$ - $8 \mathrm{MHz}$ range for the high frequency transvaginal transducer was utilized. This study was approved by the Advocate Healthcare Institutional Review Board.

\section{Statistical Analysis}

Descriptive statistics for all continuous (mean \pm SD) and categorical [N (\%)] data were calculated. Univariate analyses for comparing the dichorionic and trichorionic triplet groups were performed via Student t-tests for all continuous variables and Chi-square or Fishers Exact tests for all categorical variables. The factors that were found to be statistically significant between the dichorionic and trichorionic triplets and 3 clinically relevant predictors with statistical significant trends were included in a multivariate logistic regression model. All analyses were performed using SPSS for Windows, version 22.0 (SPSS Inc., Chicago, IL). A two-tailed p level of 0.05 was considered statistically significant in all analyses.

\section{Results}

The demographic characteristics between the dichorionic and trichorionic groups are outlined in Table 1 . Patients in both of the groups were comparable. The only demographic variables that were significantly associated with chorionicity included the number of prior preterm deliveries ( $<37$ weeks), the use of prior ART, and the number of prior intrauterine insemination (IUI) cycles $(\mathrm{p}<0.05)$. 
Table 1. Comparing trichorionic triplets and dichorionic triplets for demographic characteristics $(\mathrm{N}=172)$.

\begin{tabular}{|c|c|c|c|}
\hline Variable & Trichorionic triplets $N=119$ & Dichorionic triplets $N=53$ & p-value \\
\hline Age, mean (SD) & $35.2(4.5)$ & $34.8(5.4)$ & 0.529 \\
\hline BMI, mean (SD) & $25.0(6.4)$ & $26.1(6.3)$ & 0.308 \\
\hline Gravida, mean (SD) & $1.3(1.5)$ & $1.2(1.5)$ & 0.379 \\
\hline Nulliparious & $78(65.5)$ & $29(54.7)$ & 0.176 \\
\hline Prior preterm delivery, n (\%) & $12(10.1)$ & $1(1.9)$ & 0.067 \\
\hline \multicolumn{4}{|l|}{ Race, n (\%) } \\
\hline Non-Hispanic White & $89(74.8)$ & $46(86.6)$ & \\
\hline African American & $4(3.4)$ & $2(3.8)$ & \\
\hline Hispanic & $7(5.9)$ & $1(1.9)$ & \\
\hline Asian/Pacific Islander & $12(10.1)$ & $4(7.5)$ & \\
\hline Unknown & $7(5.9)$ & - & 0.256 \\
\hline Insurance, n (\%) & $86(100)$ & $50(98.0)$ & 0.372 \\
\hline Prior ART, n (\%) & $96(80.7)$ & $30(56.6)$ & $0.001^{*}$ \\
\hline Number of prior ART, mean (SD) & $2.4(2.4)$ & $1.7(2.0)$ & 0.060 \\
\hline Number of prior IVF, mean (SD) & $0.9(1.4)$ & $0.8(1.2)$ & 0.441 \\
\hline Number of prior FET, mean (SD) & $0.2(0.6)$ & $0.4(0.9)$ & 0.277 \\
\hline Number of prior IUI, mean (SD) & $1.2(1.6)$ & $0.5(1.1)$ & $0.007^{*}$ \\
\hline
\end{tabular}

Note: When not $100 \%$, missing values were reported; SD, standard deviation.

ICSI was performed in virtually all patients that underwent IVF. The number of embryos transferred in the dichorionic triplets were $2(n=44), 3(n=6), 5(n=1)$, and for the trichorionic triplets $2(n=23), 3(n=50), 4$ $(n=8), 5(n=4), 7(n=1)$, with a mean of 2.2 vs 3 embryos transferred $(p<0.001)$. Hatching was used in $70.6 \%$ of dichorionic versus $89.5 \%$ of trichorionic IVF triplets $(\mathrm{p}=0.005)$, embryo transfer was Day 5 or 6 versus Day 3 in $88.0 \%$ versus $71.8 \%(p=0.028)$. Frozen sperm was utilized more frequently with dichorionic than with trichorionic triplets, $26.4 \%$ versus $10.9 \%(\mathrm{p}<0.010)$. Only 4 (7.5\%) of the IVF cases underwent pre-implantation genetics. Maternal and donor blood type A+ and maximal FSH during treatment was not associated with dichorionic triplet gestations (Table 2).

The results of the regression analysis are reported in Table 3. Only 127 (of 173) patients of which 83 (65.4\%) were trichorionic triplets and 44 (34.6\%) dichorionic triplets were included in the regression analyses, while the remaining patients had missing values in one or more variables of interest. Of the variables included in the analysis, only the number of embryos transferred $(\mathrm{OR}=0.13$, 95\% $0.05-0.33, \mathrm{p}<0.001)$ and number of prior frozen embryo transfers ( $\mathrm{OR}=1.89,95 \% 1.01-3.52$, $\mathrm{p}<0.045)$ were found to be statistically significant predictors of chorionicity.

Interestingly, days from embryo transfer to triplet identification by US was on average 24.8 days for trichorionic versus 27.8 days for the dichorionic gestations ( $\mathrm{p}<0.001)$. Days from first US to identification of a triplet gestation was less than 1 day in trichorionic triplets and 4.5 days for dichorionic triplets $(\mathrm{p}<0.001)$.

\section{Discussion}

The Practice Committee of the American Society of Reproductive Medicine states that the goal of infertility treatment is for each patient to have one healthy child at a time. The challenges associated with achieving that goal differ with the treatment and clinical setting. Several factors contribute to the continuing problem of higher-order multiples from fertility treatments. An increased sense of urgency leads many infertile couple to pursue more aggressive treatments involving the use of exogenous gonadotropins, or to accept the risks associated with the transfer of a greater number of embryos in IVF cycles. Inadequacy or absence of health care coverage may 
Table 2. Comparing trichorionic triplets and monochorionic triplets for clinical variables $(\mathrm{N}=172)$.

\begin{tabular}{|c|c|c|c|}
\hline Variable & Trichorionic triplets $N=119$ & Dichorionic triplets $N=53$ & p-value \\
\hline $\begin{array}{l}\text { Successful ART type } \\
\text { IUI/ovulation induction } \\
\text { IVF } \\
\text { FET }\end{array}$ & $\begin{array}{l}33(27.7) \\
64(53.8) \\
22(18.5)\end{array}$ & $\begin{array}{c}2(3.8) \\
42(79.2) \\
9(17)\end{array}$ & 0.001 \\
\hline \multicolumn{4}{|l|}{ Fetal heartbeats, n (\%) } \\
\hline 3 & $119(100)$ & $53(100)$ & $\mathrm{Na}$ \\
\hline \multicolumn{4}{|l|}{ Egg source, n (\%) } \\
\hline Autologous & $102(85.7)$ & $43(81.1)$ & \\
\hline Donor & $17(14.3)$ & $10(18.9)$ & 0.446 \\
\hline \multicolumn{4}{|l|}{ Sperm retrieval, n (\%) } \\
\hline Fresh & $106(89.1)$ & 39 (73.6) & \\
\hline Frozen & $13(10.9)$ & $14(26.4)$ & 0.010 \\
\hline PGD, n (\%) & $3(3.5)$ & $4(7.8)$ & 0.424 \\
\hline \multicolumn{4}{|l|}{ Hatching, n (\%) } \\
\hline Assisted & 77 (89.5) & $36(70.6)$ & \\
\hline Not assisted & $9(10.5)$ & $15(29.4)$ & 0.005 \\
\hline Max FSH, mean (SD) & $10.2(13.3)$ & $8.3(2.9)$ & 0.349 \\
\hline ET number, mean (SD) & $3.0(0.9)$ & $2.2(0.5)$ & $<0.001$ \\
\hline $\begin{array}{l}\text { Transfer day, n (\%) } \\
\text { Day } 3 \\
\geq \text { Day } 5\end{array}$ & $\begin{array}{l}24(28.2) \\
61(71.8)\end{array}$ & $\begin{array}{c}6(12) \\
44(88)\end{array}$ & 0.028 \\
\hline Days to $1^{\text {st }}$ US recognition, mean (SD) & $24.3(3.3)$ & $23.2(3.9)$ & 0.066 \\
\hline Days to triplet identification, mean (SD) & $24.8(3.6)$ & $27.8(4.4)$ & $<0.001$ \\
\hline $\begin{array}{l}1^{\text {st }} \text { US recognition to triplet identification, } \\
\text { mean (SD) }\end{array}$ & $0.6(2.1)$ & $4.5(4.8)$ & $<0.001$ \\
\hline
\end{tabular}

ART, assisted reproductive technique; IVF, in vitro fertilization; FET, frozen embryo transfer; IUI, intrauterine insemination; PGD, pre-implantation genetic diagnosis; US, ultrasound; FSH, follicle-stimulating hormone, ET, embryo transfer.

Table 3. Multivariate logistic regression model with odds ratios $(\mathrm{N}=127)$.

\begin{tabular}{cccccc}
\hline Variable & $\mathbf{B}^{\mathbf{s}}$ & Wald $^{\text {\% }}$ & Odds & $\mathbf{9 5 \% ~ C I}$ & \multicolumn{2}{c}{ p-value } \\
\hline Sperm retrieval & 0.85 & 1.97 & 2.33 & $0.71-7.60$ & 0.161 \\
Hatching & -0.90 & 2.47 & 0.41 & $0.13-1.25$ & 0.116 \\
ET number & -2.04 & 18.8 & 0.13 & $0.05-0.33$ & $<0.001^{\wedge}$ \\
Retrieved prior ART & -0.51 & 0.95 & 0.60 & $0.22-1.67$ & 0.329 \\
Max FSH & -0.01 & 0.31 & 0.99 & $0.94-1.04$ & 0.575 \\
Number prior FET & 0.64 & 4.01 & 1.89 & $1.01-3.52$ & $0.045^{\wedge}$ \\
BMI & 0.02 & 0.25 & 1.02 & $0.95-1.10$ & 0.617 \\
\hline
\end{tabular}

$\wedge \mathrm{p}<0.001 ; \wedge_{\mathrm{p}}<0.05 ;{ }^{\$} \mathrm{~B}=$ coefficient for the constant/intercept in the null model; ${ }^{\%}$ Wald = Wald chi-Square test. ET, embryo transfer, ART, assisted reproductive technique FSH, follicle-stimulating hormone; FET, frozen embryo transfer; BMI, body mass index.

encourage some to pursue superovulation as a less costly alternative, or to transfer more embryos for a limited number of covered IVF cycles. Lastly, competition and the ability to quote higher success rates among fertility centers may drive some clinicians to opt for superovulation of IVF earlier in treatment [12]. 
In 1998 the Society for Assisted Reproductive Technology and the American Society for Reproductive Medicine published the first practice guidelines for the maximum number of embryos to transfer in IVF cycles according to maternal age and other prognostic factors. Recommendations were subsequently adjusted based on higher IVF success rates in 1999, 2004, 2006, and 2008. A recent publication [13] estimated that by 2011, a total of $77 \%$ of triplet and higher-order births resulted from conception assisted by fertility treatments. The incidence of triplet and higher-order births increased by a factor of 6.7 from 1971 to 1998 and decreased by 29\% from 1998 to 2011. This decrease coincided with a 70\% reduction in the transfer of three or more embryos during IVF, and a 33\% decrease in the proportion of triplet and higher-order births attributable to IVF.

The majority of triplets in our study population conceived through IVF, and limiting the number of embryos transferred was also noted in our study as 67/172 patients that underwent IVF or frozen embryo transfer had 2 embryos transferred. Nevertheless, 51 of these pregnancies resulted in a dichorionic triplet gestation. It has been demonstrated that dichorionic triplets are at increased risk for earlier deliveries and have higher perinatal mortality rates compared with trichorionic triplets [1] [14]. It is therefore imperative to try to understand what factors contribute to embryo splitting in this population.

Various factors have been reported to be associated with monozygotic twinning. These include techniques in which there is zona pellucida manipulation such as ICSI or assisted hatching, and also after frozen embryo transfer or blastocyst transfer. In our study, Day 5 or 6 blastocyst transfer, frozen sperm, and non-assisted hatching were all significantly associated with dichorionic as compared to trichorionic triplets. There is also evidence to suggest that the process of freezing and thawing embryos may cause the zona to harden, and using assisted hatching can be beneficial. A 2009 Cochrane review of 4 studies with 524 patients concluded that available data was insufficient to determine whether assisted hatching increased the risk of monozygotic twinning (OR 3.26 CI 0.14 - 77.84).

A recent study looking at factors that contribute to early embryo splitting in IVF resulting in monozygotic twins noted an association with young oocyte age, extended culture, and zona pellucida manipulation [15]. We did not find young oocyte age (donor), nor assisted hatching to be associated with an increased risk of a monochorionic pair in a triplet gestation. This may be secondary to the interplay of multiple factors affecting the zona pellucida, including the timing and technique utilized in assisted hatching, and also if the zona pellucida was hardened from freezing and thawing. Since 2012 we have used the laser for hatching. Prior to that time, zona drilling was performed with acidified tyrodes solution. Presently at FCI, all frozen embryos undergo assisted hatching on Day 3, and if needed (no zona thinning), on Day 5. We are increasingly utilizing frozen embryos with transfer the following cycle secondary to evidence that suggests the supra-physiologic estradiol and progesterone levels that occur during IVF cycles may be associated with decreased endometrial receptivity [16]. There is also a growing interest in pre-implantation genetics.

Limitations of this study include its retrospective nature, which precludes specifics about technologies used, and also missing data, which limited the regression analysis. Future studies in this area should try to be specific about the techniques and timing used in order to increase our understanding of factors that increase the risk of embryo splitting. This would also increase the generalizability of study results.

Lastly, days from embryo transfer to triplet identification by US was on average 3 days shorter for trichorionic compared to dichorionic gestations. This may be due to a thinner separating membrane being more difficult to visualize [17]. However, we believe this is secondary to the delayed embryo splitting associated with and causative of monochorionic multiple gestations. Interestingly traditional teaching is that the zygote splits Days 4-8 after fertilization to produce a monochorionic, diamniotic configuration. However, we have not observed such splitting prior to Day 6 transfer.

\section{Conclusion}

In conclusion, certain assisted reproductive technologies appear to be associated with embryo splitting and a dichorionic triplet gestation. More research is needed in this area to further elucidate these associations and possible causality, as perinatal outcomes are negatively impacted by higher-order multiple gestations, particularly when harboring a monochorionic twin pair.

\section{Conflicts of Interest}

The authors have no conflicts of interest to report. 


\section{References}

[1] (2014) American College of Obstetricians and Gynecologists. Obstetrics \& Gynecology, 123, 1118-1132.

[2] Kawaguchi, H., Ishii, K., Yamamoto, R., Hayashi, S. and Mitsyda, N. (2013) Perinatal Death of Triplet Pregnancies by Chorionicity. American Journal of Obstetrics \& Gynecology, 209, 36.e1-36.e7. http://dx.doi.org/10.1016/j.ajog.2013.03.003

[3] Bajoria, R., Ward, S.B. and Adegbite, A.L. (2006) Comparative Study of Perinatal Outcome of Dichorionic and Trichorionic Iatrogenic Triplets. American Journal of Obstetrics \& Gynecology, 194, 415-424. http://dx.doi.org/10.1016/j.ajog.2005.08.009

[4] Practice Committee of Society for Assisted Reproductive Technology, Practice Committee of American Society for Reproductive Medicine (2008) Guidelines on Number of Embryos Transferred. Fertility and Sterility, 90, S163-S164. http://dx.doi.org/10.1016/j.fertnstert.2008.08.053

[5] Ortibus, E., Lopriore, E., Deprest, J., et al. (2009) The Pregnancy and Long-Term Neurodevelopmental Outcome of Monochorionic Diamniotic Twin Gestations: A Multicenter Prospective Cohort Study from the First Trimester Onward. American Journal of Obstetrics \& Gynecology, 200, 494.e1. http://dx.doi.org/10.1016/j.ajog.2009.01.048

[6] Multifetal Gestations: Twin, Triplet, and Higher Order Multifetal Pregnancies. Practice Bulletin No. 144.

[7] Geipel, A., Berg, C., Katalinic, A., Plath, H., Hansmann, M., Germer, U., et al. (2005) Prenatal Diagnosis and Obstetric Outcomes in Triplet Pregnancies in Relation to Chorionicity. BJOG, 112, 554-558. http://dx.doi.org/10.1111/j.1471-0528.2005.00627.x

[8] Ghulmiyyah, L.M., Mark, P., Tucker, M.J., Zimmermann, J.H., Eller, D.P. and Sill, E.S. (2003) Monochorionic-Triamniotic Triplet Pregnancy after Intracytoplasmic Sperm Injection, Assisted Hatching, and Two-Embryo Transfer: First Reported Case Following IVF. BMC Pregnancy Childbirth, 3, 4. http://dx.doi.org/10.1186/1471-2393-3-4

[9] Li, Y., Yang, D. and Zhang, Q. (2009) Dichorionic Quadramniotic Quadruple Gestation with Monochorionic Triamniotic Triplets after Two Embryos Transfer and Selective Reduction to Twin Pregnancy: Case Report. Fertility and Sterility, 92, 2038.e13-2038.e15. http://dx.doi.org/10.1016/j.fertnstert.2009.08.022

[10] Marcelo, F., Bos-Mikich, A., Hoher, M. and Frantz, N. (2010) Dichorionic Twins and Monochorionic Triplets after the Transfer of Two Blastocysts. Journal of Assisted Reproduction and Genetics, 27, 545-548. http://dx.doi.org/10.1007/s10815-010-9446-z

[11] Reshef, T., Fridman, D. and Grazi, R.V. (2012) Monozygotic Triplets and Dizygotic Twins Following Transfer of Three Poor-Quality Cleavage Stage Embryos. Case Reports in Obstetrics and Gynecology, 2012, Article ID: 763057.

[12] Practice Committee of American Society for Reproductive Medicine (2012) Multiple Gestation Associated with Infertility Therapy: An American Society for Reproductive Medicine Practice Committee Opinion. Fertility and Sterility, 97, 825. http://dx.doi.org/10.1016/j.fertnstert.2011.11.048

[13] Kulkarni, A.D., Jamieson, D.J., Jones Jr., H.W., et al. (2013) Fertility Treatments and Multiple Births in the United States. New England Journal of Medicine, 369, 2218. http://dx.doi.org/10.1056/NEJMoa1301467

[14] Spencer, J.V. (2009) Perinatal and Neonatal Outcomes of Triplet Gestations Based on Placental Chorionicity. American Journal of Perinatology, 26, 587-590. http://dx.doi.org/10.1055/s-0029-1220776

[15] Knopman, J.M., Krey, L.C., Oh, C., Lee, J., McCaffrey, C. and Noyes, N. (2014) What Makes Them Split? Identifying Risk Factors That Lead to Monzygotic Twins after in Vitro Fertilization. Fertility and Sterility, 102, 82-89. http://dx.doi.org/10.1016/j.fertnstert.2014.03.039

[16] Shapiro, B.S., Daneshmand, S.T., Restrepo, H., Garner, F.C., Aguirre, M. and Hudson, C. (2013) A Matched Cohort Comparison of Single Embryo Transfers in Fresh and Frozen Thawed Embryo Transfer Cycles. Fertility and Sterility, 99, 389-392. http://dx.doi.org/10.1016/j.fertnstert.2012.09.044

[17] Doubilet, P.M. and Benson, C.B. (1998) “Appearing Twin”: Undercounting of Multiple Gestations on Early First Trimester Sonograms. Journal of Ultrasound in Medicine, 17, 199-203. 